\title{
OUVIDORIA DA SECRETARIA DE ESTADO DE SAÚDE DO RIO DE JANEIRO: BOAS PRÁTICAS NA ÁREA DA SAÚDE
}

\author{
Evelyn Silvano da Silva ${ }^{1}$ \\ Luana dos Santos Pimentel ${ }^{2}$ \\ Márcia Lopes Silva ${ }^{3}$
}

\begin{abstract}
Resumo
A experiência exitosa descrita no presente artigo teve como principal objetivo estruturar um monitoramento de excelência das Ouvidorias Descentralizadas pertencentes à estrutura da Secretaria de Estado de Saúde do Rio de Janeiro. Para atingir o objetivo proposto, a equipe da Ouvidoria da SES utilizou como metodologia a realização de ciclos de reuniões com os serviços de Ouvidoria dos Hospitais da SES/RJ, acompanhamento contínuo por amostragem do sistema OuvidorSUS - utilizado pelas Ouvidorias Descentralizadas para a inserção das manifestações cidadãs -, emprego da estratégia "Ouvidoria Itinerante", monitoramento dos relatórios gerenciais e elaboração de ferramentas eficazes de gestão da informação, assim como foram ofertadas atividades permanentes de qualificação profissional com o intuito de propiciar as condições adequadas para o pleno desenvolvimento laborativo dos funcionários que atuam nas Ouvidorias Descentralizadas, dependentes administrativa, hierárquica e tecnicamente da Ouvidoria da SES/RJ. Como resultado, temos um panorama completo e substancial no banco de dados da Ouvidoria da SES/RJ que possibilita o real monitoramento das Ouvidorias Descentralizadas.
\end{abstract}

Palavras-chave: Monitoramento. Ouvidoria. Controle Social. Capacitação. Ouvidoria Itinerante.

1 Graduada em Serviço Social e Pós-Graduada em Saúde Mental - IPUB/UFRJ. (evelyn.silvano@saude.rj.gov.br)

2 Graduada em Serviço Social pela Universidade Federal do Rio de Janeiro e Pós-Graduada em Segurança Pública e Cidadania pela Universidade Cândido Mendes. (luana.pimentel@saude.rj.gov.br)

3 Ouvidora SES RJ. Psicóloga. Especialista e Mestranda em Políticas Públicas e Direitos Humanos. (marcia. lopes@saude.rj.gov.br) 


\begin{abstract}
The successful experience described in this article had as main objective to structure a monitoring of excellence of the Decentralized Ombudsmen belonging to the structure of the Secretary of State for Health of Rio de Janeiro. In order to achieve the proposed objective, the SES Ombudsman team used the methodology of meeting cycles together with the Ombudsman services of the SES/RJ Hospitals, continuous monitoring by sampling of the OuvidorSUS system - used by the Decentralized Ombudsmen for the insertion of the citizen demonstrations -, use of the "Ombudsman Itinerant" strategy, monitoring of management reports and development of effective information management tools as well as permanent professional qualification activities with the purpose of providing adequate conditions for the full development of the employees in the Ombudsman's Offices, administrative, hierarchical and technically dependent to the Ombudsman's Office SES/RJ. As a result, we have a complete and substantial overview of the database of the SES/RJ Ombudsman's Office that allows the real monitoring of the Decentralized Ombudsmen.
\end{abstract}

Keywords: Monitoring. Ombudsman. Social Control. Training. Traveling Ombudsman. 


\section{INTRODUÇÃO}

A Ouvidoria-Geral e as Ouvidorias Descentralizadas pertencem à estrutura da Secretaria de Estado de Saúde do Rio de Janeiro (SES/RJ) e compõem a Ouvidoria da Saúde. Todas as Ouvidorias Descentralizadas estão subordinadas hierárquica, administrativa e tecnicamente à Ouvidoria-Geral (Art. $4^{\circ}$ Caput e $\S 2^{\circ}$ - Resolução SES 207/2011).

A Ouvidoria tem papel relevante na identificação das necessidades e demandas sociais, tanto na dimensão coletiva quanto na individual, transformando-as em suporte estratégico para a tomada de decisões no campo da gestão.

A Ouvidoria deve estimular o protagonismo do cidadão: entende-se que é através do indivíduo que se inicia a resolução ou correção de um processo que afeta uma coletividade.

Para isso, são necessários instrumentos adequados à realização de um trabalho que prime pela resolubilidade e transparência, essenciais a um serviço de excelência em Ouvidoria.

A Ouvidoria possui como principal atribuição o acolhimento das manifestações dos cidadãos beneficiários dos serviços prestados pelo Sistema Único de Saúde (SUS) em âmbito estadual. Recebe manifestações classificadas em: reclamações, informações, denúncias, solicitações, sugestões e elogios.

\section{PLANO ESTADUAL DE SAÚDE (2016-2019)}

O Sistema Único de Saúde utiliza-se de instrumentos de planejamento para aperfeiçoar suas ações. Inicialmente é construído o Plano Estadual de Saúde (PES), de periodicidade quadrienal, onde são traçadas as macrodiretrizes para o planejamento das ações a serem desenvolvidas pela Secretaria de Estado de Saúde. As metas que deverão ser alcançadas a cada ano são traçadas no documento denominado Programação Anual de Saúde (PAS), e também anualmente os resultados são descritos no Relatório Anual de Gestão (RAG).

A Ouvidoria da SES está inserida no Plano Estadual de Saúde, no Eixo III - Gestão em Saúde na terceira diretriz, qual seja: "Fortalecer a gestão do SUS, de modo a melhorar e aperfeiçoar a capacidade resolutiva das ações e serviços prestados à população". Inscrita no objetivo: "Ampliar e fortalecer a participação popular e o controle social, tendo como estratégia o fortalecimento da política de gestão estratégica e participativa com vistas à promoção da equidade e participação social".

Uma das metas na qual a Ouvidoria está inscrita visa "Apoiar tecnicamente $100 \%$ dos serviços de Ouvidoria municipais", pertencendo ao indicador de "Percentual dos serviços de Ouvidoria municipais apoiados tecnicamente". A meta de "Realizar Ouvidoria Itinerante em 100\% das unidades de saúde da SES/RJ" possui o indicador de "Percentual das unidades de saúde da SES/RJ em que foram realizadas Ouvidorias Itinerantes". Há, também, a meta "Apoiar tecnicamente 100\% das Ouvidorias Descentralizadas pertencentes à estrutura da SES/RJ". O indicador em questão é o "Percentual das unidades de saúde da SES/RJ em que foram realizadas Ouvidorias Itinerantes".

O referido documento de planejamento traz ainda o objetivo da Ouvidoria de "Promover o desenvolvimento institucional da SES/RJ com vistas à integração das suas áreas e à melhoria da 
execução das ações planejadas", posto na estratégia da "Valorização da percepção do cidadão com vistas ao aperfeiçoamento das ações de saúde da SES/RJ, através da análise dos dados da Ouvidoria". A meta correspondente ao alcance desse objetivo é de "Disseminar 100\% das demandas acolhidas pela Ouvidoria às áreas técnicas competentes", tendo como indicador correspondente o "Percentual das demandas acolhidas pela Ouvidoria disseminadas às áreas técnicas competentes".

A definição de estratégias no Plano Estadual de Saúde faz-se de grande importância para programar, acompanhar, realizar e verificar a eficácia, eficiência e a efetividade das ações pelas quais cada Ouvidoria da SES/RJ é responsável.

O objetivo do presente artigo é demonstrar os resultados obtidos com a meta de apoio técnico às Ouvidorias Descentralizadas, como também a experiência de realização de Ouvidorias Itinerantes, ambas atividades inseridas no Plano Estadual de Saúde da SES/RJ.

\subsection{Monitoramento das Ouvidorias Descentralizadas}

As Ouvidorias Descentralizadas são serviços que funcionam nas unidades hospitalares pertencentes à estrutura administrativa da Secretaria de Estado de Saúde do Rio de Janeiro. Atualmente existem 29 serviços em atividade, que realizaram o quantitativo de atendimentos conforme descrito no gráfico 1 :

\section{GRÁFICO 1 - Atendimentos Ouvidorias Descentralizadas - 2015 a julho de 2018}

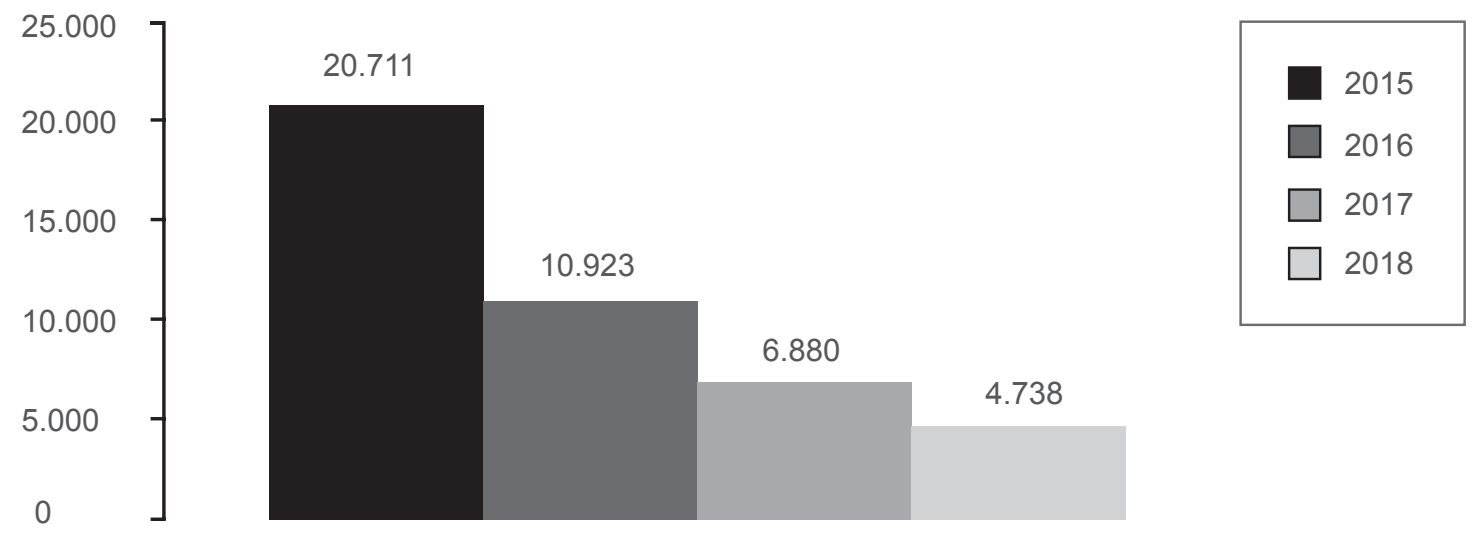

O monitoramento das Ouvidorias Descentralizadas está inscrito na meta do Plano Estadual de Saúde de "Apoiar tecnicamente 100\% das Ouvidorias Descentralizadas pertencentes à estrutura da SES/RJ".

No âmbito da base legal das Ouvidorias do SUS, a política que possui amplo destaque é a Política Nacional de Gestão Estratégica e Participativa. Ela foi criada com o desafio de promover e estimular a formulação e a construção de políticas públicas de saúde de forma participativa, fortalecendo assim o controle social. Para o alcance dessa proposta, tem sido desenvolvida a criação de 
espaços públicos e coletivos para o exercício do diálogo e da pactuação de ações para o desenvolvimento da melhoria da gestão do Sistema Único de Saude, dando forma e estrutura à participação da comunidade como diretriz organizativa do sistema (BRASIL, 2010).

Como componente desta Política, a Ouvidoria em Saúde constitui uma ferramenta estratégica e democrática de comunicação entre o cidadão e os gestores do SUS.

\subsection{Ciclos de Reuniões}

A Ouvidoria da SES/RJ, como referência técnica para as Ouvidorias Descentralizadas, presta todo o apoio visando o pleno desenvolvimentos dos serviços de Ouvidoria nas respectivas unidades de saúde. Como metodologia, são realizados periodicamente ciclos de reuniões em que as unidades hospitalares que possuem serviço de Ouvidorias Descentralizadas são divididas, segundo o perfil de gestão, em três grupos. Essa formatação de encontros por ciclos deu-se a partir do ano de 2015. Até o presente momento, realizaram-se oito ciclos de reuniões. Não há uma periodicidade definida para esses encontros. Os ciclos ocorrem de acordo com a necessidade apresentada pelas Ouvidorias Descentralizadas, sendo no mínimo dois ciclos anuais.

Em todos os ciclos de reuniões com as Ouvidorias Descentralizadas, foi aplicado o instrumento de "Avaliação de Reação", com objetivo de medir a satisfação dos participantes. Tal ferramenta foi sugerida pela Assessoria de Qualidade da SES em 2008. A Avaliação de Reação é composta pelos itens de: Clareza no(s) objetivo(s) do evento, Metodologia, Motivação, Conteúdo/Assunto da Reunião, Duração da Reunião, Infraestrutura, Atendimento da Equipe (no item 1); Desempenho da Equipe, Qualidade dos Recursos Operacionais, Integração, Comunicação, Domínio do Conteúdo (no item 2); e ainda a pergunta: "De modo geral, como você classifica a reunião?"(no item3). Os dados colhidos são posteriormente tabulados por evento realizado e depois consolidados em tabela.

No gráfico 2, demonstramos a avaliação dos participantes dos ciclos de reunião realizados em 2017:

\section{GRÁFICO 2 - Classificação Geral dos Ciclos de Reunião}

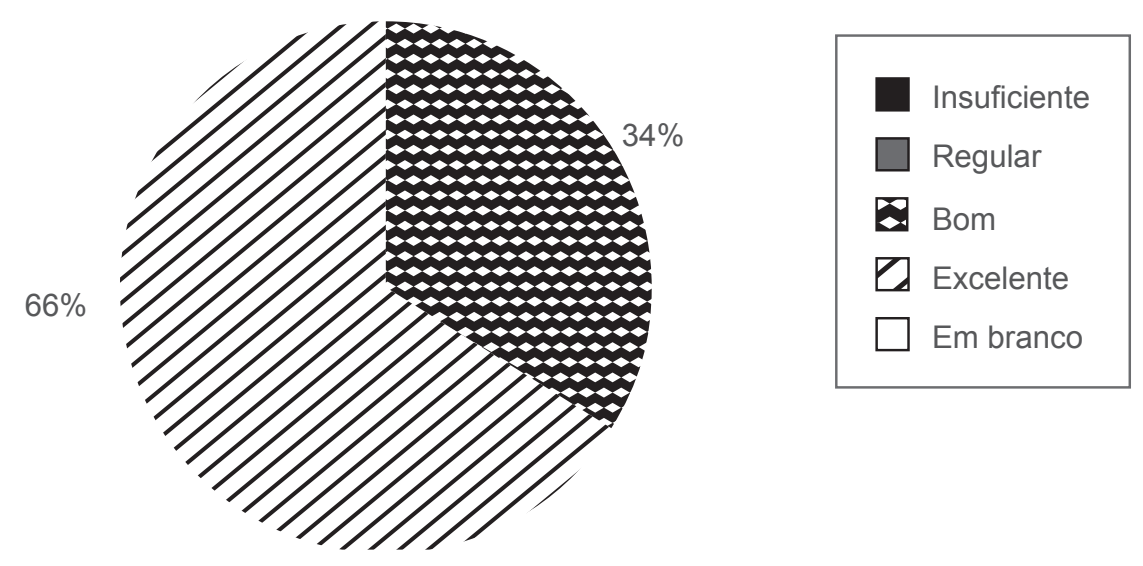


Ressalta-se que, de acordo com as necessidades apresentadas pelas Ouvidorias Descentralizadas, realizam-se reuniões integrando todas as unidades em um único encontro, com o objetivo de propiciar o enriquecimento, fortalecimento das relações e troca de experiências entre os Ouvidores lotados nessas Ouvidorias. Alguns encontros são temáticos e contam com convidados externos que possam contribuir para a adequada qualificação dos Ouvidores. Em determinados momentos, convocamos os Ouvidores Coordenadores a apresentarem suas experiências exitosas.

Na medida em que ocorre uma troca entre os profissionais das Ouvidorias Descentralizadas, esse espaço apresenta-se como um forte potencializador para o fortalecimento do processo de trabalho, vislumbrando a sua melhora ao instrumentalizar a gestão por meio dos relatórios gerenciais produzidos pelas Ouvidorias.

Os ciclos de reuniões com as Ouvidorias Descentralizadas foram pensados com a finalidade de possibilitar uma troca de informações que proporcionasse a construção de conhecimento e qualificação entre as Ouvidorias e, consequentemente, a melhoria e o aprimoramento dos serviços prestados aos cidadãos no que compete ao setor, tendo em vista a compreensão desses espaços como "um lugar de interação e, consequentemente, de desenvolvimento da aprendizagem colaborativa, uma vez que esta é pautada na interação e na participação ativa/colaborativa de todos os envolvidos no processo de ensino aprendizagem" (PALLOF e PRATT, 2002).

As reuniões com as Ouvidorias Descentralizadas constituem-se assim "um espaço privilegiado para a troca de ideias onde ocorre o entrelaçamento de muitas vozes que constroem e desconstroem, questionam e respondem" (OKADA, 2006, apud BARROS; SOUZA, 2011, p. 384), representando nesta perspectiva um terreno fértil de fortalecimento de princípios democráticos de participação e controle social, na medida em que visam uma releitura constante do fazer profissional dos Ouvidores, que têm como principais questões as demandas dos usuários dos serviços de saúde que atendem.

\subsection{Ferramentas Desenvolvidas para Propiciar a Gestão da Informação nas Ou- vidorias Descentralizadas}

Durante todo o período em que a Ouvidoria da SES realiza o monitoramento dos serviços de Ouvidorias Descentralizadas das unidades hospitalares, a equipe da Ouvidoria da SES acompanha os Relatórios Gerenciais que os serviços produzem. Tal monitoramento consiste em uma avaliação minuciosa tanto da parte quantitativa como da parte qualitativa de tal ferramenta. Os parâmetros para monitorar as conformidades e não conformidades na elaboração de tais relatórios são:

1. Consistência nos dados - é verificada a coerência das informações apresentadas nas tabelas, planilhas e gráficos e há a adição do indicador "Resolubilidade Específica". Tal indicador mensura as manifestações classificadas como reclamação, solicitação e denúncia.

2. Elaboração de Análise Qualitativa - procura-se estimular as Ouvidorias para que continuem desenvolvendo reflexões sobre os dados apresentados no período em questão 
no relatório, de forma a fomentar a contribuição da Ouvidoria na melhoria da gestão da unidade hospitalar.

Por meio do acompanhamento desenvolvido ao longo dos anos, o suporte técnico da Ouvidoria da SES notou que havia a necessidade de substanciar as informações contidas na análise crítica. Para tanto, foi elaborado um modelo com o intuito de servir de guia para a confecção das análises críticas. Tal modelo segue anexado a este artigo.

A partir das análises dos relatórios gerenciais produzidos pelas Ouvidorias Descentralizadas, é elaborado periodicamente um panorama geral quantitativo e qualitativo dos atendimentos registrados e das análises críticas com base nas questões levantadas pelos Ouvidores Coordenadores.

Desde o ano de 2015, as Ouvidorias Descentralizadas lançam mão do sistema disponibilizado pelo Departamento de Ouvidoria-Geral do SUS (DOGES), órgão ligado ao Ministério da Saúde, qual seja: o sistema OuvidorSUS. A referida plataforma online é oferecida pelo DOGES gratuitamente e é utilizado pela Rede de Ouvidorias do SUS existente no plano nacional. As Ouvidorias Descentralizadas são capacitadas no sistema OuvidorSUS pela equipe da Ouvidoria da SES de acordo com a necessidade de qualificação apresentada. A inserção de manifestações no referido sistema é monitorada por profissionais da equipe da SES, e esse monitoramento é realizado por amostragem - são analisados $10 \%$ do total de manifestações inseridas por cada equipe de Ouvidoria Descentralizada, e tal controle é realizado mensalmente.

Para além das capacitações no sistema OuvidorSUS, a Ouvidoria da SES presta respaldo teórico metodológico que oferece a qualificação necessária para que os Ouvidores possam atuar nos serviços das Ouvidorias Descentralizadas. Dessa forma, são realizados diversos encontros de aprimoramento com o intuito de estabelecer a educação permanente dos profissionais lotados nas unidades hospitalares. Durante o ano de 2017 foram realizados oito eventos no total, entre reuniões, capacitações e o $3^{\circ}$ Seminário de Ouvidorias do SUS no estado do Rio de Janeiro, reunindo 156 profissionais.

Ressalta-se que a SES RJ possui contratos com Organizações Sociais que realizam a gestão operacional de unidades hospitalares. No intuito da manutenção dos serviços de Ouvidoria foram inseridos nos contratos de gestão os critérios de implantação das Ouvidorias Descentralizadas, bem como o indicador de resolubilidade no rol de indicadores que são acompanhados pelos setores de fiscalização da SES. Conforme o indicador de resolubilidade, a unidade hospitalar deve apresentar um percentual de demandas resolvidas/respondidas referente às demandas classificadas como reclamação, solicitação e denúncia. Acreditamos que o acompanhamento desse indicador possibilita o envolvimento de toda a instituição nas questões pertinentes à percepção do cidadão que está utilizando o serviço. O método de cálculo apresenta-se da seguinte forma:

\section{Total de manifestações resolvidas \\ Total das reclamações, solicitações e denúncias recebidas $x 100$}

Meta: $>=90 \%$ 


\section{OUVIDORIA ITINERANTE}

Em conformidade com a meta inscrita no PES de "Realizar Ouvidoria Itinerante em 100\% das unidades de saúde da SES/RJ", objetiva-se desenvolver estratégias de Ouvidoria Itinerante com o intuito de promoção e ampliação desse canal de comunicação, favorecendo o encontro do cidadão com a gestão estadual do SUS. Seus objetivos específicos:

- Realizar o acolhimento das manifestações em unidades (fixas e móveis) da estrutura SES que possuam ou não Ouvidorias Descentralizadas em funcionamento.

- Acolher as manifestações das populações com situações de vulnerabilidade.

Para tanto, utiliza-se uma equipe de aproximadamente quatro técnicos que fazem a divulgação do serviço de Ouvidoria da Secretaria de Estado de Saúde do Rio de Janeiro, o acolhimento das manifestações quando necessário e a disseminação de informações em saúde.

No quadro que se segue, apresentam-se algumas informações sobre as atividades de Ouvidoria Itinerante realizadas no período de 2015 a 2018:

\section{QUADRO 1 - Ouvidorias Itinerantes realizadas no período de 2015 a 2018}

\begin{tabular}{|l|l|l|}
\hline AÇÃO & DATA & $\begin{array}{l}\text { QTD. } \\
\text { REGISTROS }\end{array}$ \\
\hline Ouvidoria Itinerante - Central do Brasil & $15 / 10 / 2015$ & 104 \\
\hline Ouvidoria Itinerante - Nova Iguaçu & $17 / 11 / 2015$ & 80 \\
\hline Ouvidoria Itinerante - São Gonçalo & $24 / 11 / 2015$ & 71 \\
\hline Ouvidoria Itinerante - Niterói & $11 / 12 / 2015$ & 47 \\
\hline Ouvidoria Itinerante SES - Hospital Estadual Adão Pereira Nunes & $17 / 08 / 2017$ & 6 \\
\hline Ouvidoria Itinerante SES - Hospital Estadual Getúlio Vargas & $22 / 08 / 2017$ & 4 \\
\hline Ouvidoria Itinerante SES - Central do Brasil & $12 / 09 / 2017$ & 18 \\
\hline Ouvidoria Itinerante SES - Hospital Estadual Azevedo Lima & $20 / 09 / 2017$ & 5 \\
\hline $\begin{array}{l}\text { Ouvidoria Itinerante SES - Hospital Estadual da Mulher Heloneida } \\
\text { Studart }\end{array}$ & $26 / 09 / 2017$ & 1 \\
\hline Ouvidoria Itinerante SES - Hospital Estadual Alberto Torres & $05 / 10 / 2017$ & 5 \\
\hline $\begin{array}{l}\text { Ouvidoria Itinerante SES - Instituto Estadual de Cardiologia Aloysio } \\
\text { de Castro }\end{array}$ & $09 / 11 / 2017$ & 4 \\
\hline Ouvidorias Públicas - Largo da Carioca & $16 / 03 / 2018$ & 2 \\
\hline TOTAL & & 347 \\
\hline
\end{tabular}

\section{CONSIDERAÇÕES FINAIS}

A descrição da experiência realizada pela Ouvidoria da Secretaria de Estado de Saúde do Rio de Janeiro teve o intuito de divulgar, para os setores que prestam serviços de Ouvidoria e/ou pes- 
soas interessadas pela temática, o trabalho desenvolvido como uma forma exitosa de construção de ferramenta capaz de fomentar o controle e a participação social, propiciando a qualificação dos atores envolvidos nos serviços de Ouvidoria. Ainda que seja um modelo eficaz, seu aperfeiçoamento será contínuo e dependente da participação dos sujeitos que a constituem.

As atividades de monitoramento permitem aos Ouvidores, a partir do levantamento e socialização das experiências do serviço prestado, reforçar o papel de Ouvidor desenvolvido com a população, proporcionando-lhes o reconhecimento mútuo como contribuintes para a melhoria dos serviços públicos de saúde de suas unidades. Essa troca garante, portanto, o conhecimento do que é vivenciado pelos outros profissionais que atuam no mesmo papel, mas em condições diferentes, levando-se em conta as especificidades de cada hospital, trazendo com isso o fortalecimento da Ouvidoria como ferramenta fomentadora da participação e do controle social.

A partir dos indicadores obtidos com a sistematização dos dados coletados por meio da Avaliação de Reação, verifica-se que os participantes dos ciclos de reuniões, em sua grande maioria, classificaram os encontros como "excelente", com isso nota-se que o índice de satisfação em relação a essa atividade encontra-se alinhado à busca pela Ouvidoria-Geral da SES/RJ do aprimoramento contínuo dos seus processos e com a prestação de um serviço de qualidade.

A pretensão aqui foi a de socializar os modelos escolhidos, elaborados e executados, pensando na possibilidade dessa experiência inspirar outras Ouvidorias que pretendam monitorar sua rede de Ouvidorias com o mesmo intuito: o de contribuir efetivamente para a consolidação de um serviço público cada vez mais fortalecido que propicie a excelência para todos, utilizando-se para isso do serviço da Ouvidoria como ferramenta contribuidora de tal processo.

Cada vez mais a ampliação de espaços participativos permite ao cidadão auxiliar na formulação de políticas públicas, em especial no sistema público de saúde que possui nas suas normativas fundantes a participação popular como um de seus princípios. A experiência das Ouvidorias Itinerantes leva o atendimento às manifestações para locais onde não existe o acesso ao serviço e demonstra que a Ouvidoria-Geral da SES atua em conjunto com as Ouvidorias Descentralizadas. Assim, em um momento de recrudescimento das ações públicas de saúde, a atuação das Ouvidorias é de vital importância para a participação e humanização dos serviços.

Segundo Sérgio AROUCA (1986), "É sempre fundamental estar trabalhando com utopias; entretanto, numa determinada conjunção de forças políticas, essas propostas ganham força e acabam se tornando realidade". Por isso, o esforço empreendido para o fortalecimento dos princípios de participação e controle social democrático através da experiência de efetivação das atividades de monitoramento aqui descritas busca ser uma contribuição para a construção incessante de uma democracia exercida em sua plenitude.

\section{REFERÊNCIAS}

AROUCA, SÉRGIO. Revista Tema, n. 7, ag. 1986. Disponível em: http://www6.ensp.fiocruz.br/radis/revista-radis/03/ comunicacao_e_saude/ha-16-anos-arouca-ja-alertava-para-necessidade-de-uma-reforma-s.

BRASIL. MINISTÉRIO DA SAÚDE. Secretaria de Gestão Estratégica e Participativa. Política Nacional de Gestão Estratégica e Participativa no SUS - ParticipaSUS / Ministério da Saúde, Secretaria de Gestão Estratégica e Participativa. 2. ed. Brasília: Editora do Ministério da Saúde, 2010. 
Constituição da República Federativa do Brasil. Brasília: Senado Federal, 1988.

MINISTÉRIO DA SAÚDE. Secretaria de Gestão Estratégica e Participativa. Departamento de Ouvidoria-Geral do SUS. Ouvidoria Ativa do SUS: ampliando a escuta e o acesso à informação do cidadão. Brasília: Ministério da Saúde, 2014.

BARROS, Juliana de Carvalho; SOUZA, Patricia Nora. Práticas discursivas de uma tutora em fóruns de discussão online. In: Veredas on line - Temática. Programa de Pós-Graduação em Linguística. UFJF. Juiz de Fora. 2011, p. 383-397.

DALLARI, D. A. Sociedade, Estado e direito: caminhada brasileira rumo ao século XXI. In: MOTA, C. G. (Org.). Viagem incompleta - a experiência brasileira (1500-2000): a grande transação. São Paulo: SENAC, 2000.

Dicionário Priberam da Língua Portuguesa, 2008-2013. Disponível em: http://www.priberam.pt/dlpo/Fórum. Acesso em: 24 jun. 2014.

FALEIROS, Vicente de Paula; VASCONCELLOS, Luis Carlos Fadel de; SILVA, Jacinta de Fátima Senna da; SILVEIRA, Rosa Maria Godoy. A construção do SUS: histórias da reforma sanitária e do processo participativo. Brasília: Ministério da Saúde, 2006. 300 p.

GARCIA, Ronaldo Coutinho. Subsídios para organizar avaliações da ação governamental. Texto para discussão $\mathrm{n}^{\circ}$ 776. Brasília: Ipea, 2001.

LUNA, Francisco Vidal; KLEIN, Herbert. Desigualdade e indicadores sociais no Brasil. In: O sociólogo e as políticas públicas: Ensaios em homenagem a Simon Schwartzman. SCHWARTZMAN, Luisa Farah; SCHWARTZMAN, Isabel Farah; SCHWARTZMAN, Felipe Farah; SCHWARTZMAN, Michel Lent (Org.). Rio de Janeiro: Ed. FGV, 2009. p. 97 116.

PALHA, Pedro F.; VILLA, Tereza Cristina Scatena. A descentralização como eixo norteador na reorganização e operacionalização dos princípios do Sistema Único de Saúde. In: Revista Escolar de Enfermagem. USP, p. 19-26, 2003.

PALLOFF, R. M.; PRATT. K. Construindo comunidades de aprendizagem no ciberespaço. Tradução de Vinícius Figueira. Porto Alegre: Artmed, 2002.

ROLIM, Leonardo Barbosa; CRUZ, Rachel de Sá Barreto; SAMPAIO, Karla Jimena Araújo de Jesus. Participação popular e o controle social como diretriz do SUS: uma revisão narrativa. In: Saúde em Debate. Rio de Janeiro, v. 37, n. 96, p. $139-147$, jan/mar 2013

SOUZA, Aline Ferreira de; RAMALHO, Rosângela Pallano. Controle social do SUS: Desafios para uma gestão democrática e participativa. 24 p. Curso de especialização em gestão pública municipal - Paraíba, UFPB, 2011. 